and hence, from the previous Sections, covariant derivatives which transform like $\Psi$-fields with $V_{i}(M)$ given by (19) can be written as

$$
\left(D_{ \pm}^{\mu} M_{j}\right)=S_{j k}(M) d_{ \pm}^{\mu} M_{k}
$$

where $S$ is the tensor which according to (19) and (20) yields $V_{i}(M)\left({ }^{19,20}\right)$.

$\left({ }^{18}\right)$ The use of covariant derivatives to write down effective Lagrangians (Weinberg's approach) can $b$; seen clearly to be equivalent to an approach such as used at the $K_{2}$ level by CHANG and GÜRSEY ( $\left.{ }^{1}\right)$. It seems that Weinberg's approach is often simpler in practice.

$\left({ }^{20}\right)$ The $K_{n}$ invariant meson-kinetic-energy term is proportional to

$$
\left(D^{\mu}+M_{j}\right)\left(D^{\mu}+M_{j}\right)=\left(d^{\mu}+M_{j}\right)\left(d^{\mu}+M_{j}\right)=\left(d^{\mu}-M_{j}\right)\left(d^{\mu}-M_{j}\right) .
$$

\title{
Nucleon Eloctromagnetic Form Factors and Vector-Boson Resonances.
}

\author{
V. WATAghiN \\ Istituto di Fisica Teorica dell'Università - Torino \\ Istituto Nazionale di Fisica Nucleare - Sezione di Torino \\ (Nuovo Cimento, 54 A, $840(1968)$ )
}

There is an error in the last formula of p. 846 which should read

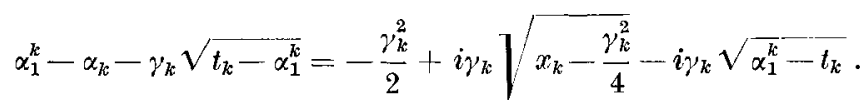

